\title{
Ameliorating effects of okra (Abelmoschus esculentus) seed oil on hypercholesterolemia
}

\author{
Moazzam Rafiq KHAN ${ }^{1}$, Maria BATOOL ${ }^{1}$, Rai Muhammad AMIR ${ }^{2 *}$ (), Asim SHABBIR ${ }^{1}$, Farzana SIDDIQUE ${ }^{3}$, \\ Rana Muhammad ADIL ${ }^{1}$, Kashif AMEER ${ }^{2}$, Ahmad DIN ${ }^{1}$, Allah RAKHA ${ }^{1}$ Aayesha RIAZ ${ }^{4}$ Farrukh FAIZ ${ }^{5}$
}

\begin{abstract}
Lifestyle associated disorders have always been a hazard for the advancement of chronic diseases. Therefore, use of natural dietary sources is proved to be safe for reducing the occurrence and management of chronic diseases. Okra along with its various parts has been used for the treatment of several diseases. The present study was done to examine the ameliorating effect of okra seed oil on hypercholesterolemic rats. Spargue Dawley Rats were randomly divided into five groups fed with selected experimental diets for a period of 30 days. After inducing hypercholesterolemia, the rats in treatment groups were fed with $6 \%, 12 \%$ and $18 \%$ okra seed oil respectively mixed with their normal diet. Decrease in cholesterol and LDL were observed in group 2, 3 and 4. Increase in HDL was also observed in group 2, 3 and 4 from $24.33 \pm 1.29$ to $25.33 \pm 1.29,23 \pm 2.94$ to $25 \pm 2.94$ and from $23.67 \pm 1.29$ to $26.67 \pm 1.29$ at day 28 . A decrease pattern was also observed in triglycerides in group 2,3 and 4 from $74 \pm 2.22$ to $72 \pm 2.22,72 \pm 1.63$ to $68 \pm 1.63$ and from $74 \pm 3.11$ to $66.67 \pm 2.45$ at day 28 . Okra seed oil has the potential to ameliorate hypercholesterolemia. This vegetable can be a part of medical nutrition therapy.
\end{abstract}

Keywords: okra; cholesterol; hypercholesterolemia; diet; lipoproteins.

Practical Application: Okra has significant healing properties to treat hypercholesterolemia.

\section{Introduction}

Lifestyle linked disorders have been a threat for the drastic outcomes in later stages of life. The prevalence of these disorders is currently increasing more in developing countries as compared to developed world due to abrupt transition in the eating habits and lifestyle pattern. Adequate and balanced intake of nutrients is always been a key of healthy life. In the present era, refined carbohydrates and processed foods are more preferred to consume upon organic products. Utilization of energy with these caloric dense foods has led to the advancements of metabolic disorders (Misra et al., 2018). Among non-communicable diseases, heart disease has now gained a great attention of health investigators. According to Australian Bureau of Statistics and Cancer Biology, heart diseases are one of the two top reasons of death in Australia and UK, the other is cancer. Various studies concluded that obesity, diabetes, stress, smoking, raised serum low density lipoprotein cholesterol, hypertension and lack of exercise are the contributing factors in the development of heart diseases (Nahar et al., 2013).

One of the blossoming species of Malvaceae family is okra (Abelmoschus esculentus). The origin of okra was from Ethiopia. Then it was propagated to tropical and sub-tropical regions including East and South Asia. Okra has been cultivated as an oil seed crop particularly in regions that are not suitable for cotton seed production and used as an alternative of cotton seed oil. Okra seed oil contains important fatty acids essential for human nutrition. Various researches claimed the cholesterol lowering ability of okra seed oil. About $20-40 \%$ of oil is produced from okra seeds (Gemede et al., 2015). The quantity of oil depends on seed variety, climate conditions, temperature and extraction method, for example the locally developed Pakistani variety (Sabz Pari) had lower oil content i.e., 11.72\% (Soares et al., 2012). In brief, okra has functional and nutraceutical properties. The present study was designed for the bio evaluation of okra seed oil with reference to hypercholesterolemia and aimed at following objectives: 1) extraction of oil from okra (Abelmoschus esculentus) seeds of local variety 2) utilization of okra seed oil in evaluating its hypocholesterolemic effects in rat model.

\section{Materials and methods}

The current study was held in University of Agriculture, Faisalabad. Vegetable and oil related research sections were used to evaluate different parameters of this study. The antihypercholesterolemic effect of okra (Abelmoschus esculentus) seed oil was studied by efficacy trials on Sprague Dowley rats. Materials and protocols used in this study are as follows:

\subsection{Attainment of raw material}

The seeds of local variety of okra named "sabaz pari" were purchased from Ayub Agriculture Research Institute, Faisalabad. 
Reagents and standards were procured from Merck (Merck KGaA, Darmstadt, Germany) and Sigma-Aldrich (Tokyo, Japan). Moreover, diagnostic kits of Cayman Chemical (Cayman Europe, Estonia), Bioassay (Bioassays chemical Co. Germany) and Sigma-Aldrich were used.

\subsection{Sample preparation}

\section{Proximate analysis of seeds}

For proximate analysis, three replicates for each sample were prepared. These analyses were performed based on the guidelines of AOAC (Association of Official Analytical Chemists, 2016). Powdered seeds were examined for subsequent parameters: 1 ) moisture content (\%), 2) crude fat, 3) crude protein, 4) crude fiber.

\subsection{Oil extraction}

After procurement of the okra seeds from Ayub Agriculture Research Institute Faisalabad, the seeds were cleaned to remove the dirt particles. Oil was extracted mechanically from cold press oil machine based on the method mentioned by Topkafa (2016). After extraction, the oil was analyzed through physiochemical analysis.

\subsection{Physiochemical analysis of extracted oil}

The oil obtained from mechanical extraction was further analyzed to study the following physiochemical properties:

\section{Saponification value}

The saponification value of okra seed oil was measured by the method mentioned in AOCS Method cd 3-25 (Association of Official Analytical Chemists, 2016).

\section{Peroxide value}

Peroxide value of oil indicates the presence of peroxides in it. Greater the concentration of these oxides indicates compromised quality of that oil. To find the peroxide value of okra seed oil, the instructions in the method of AOCS (American Oil Chemists' Society, 1998) were followed.

\section{Acid value}

It was determined by acid base titration.

\section{Specific gravity}

Based on the instructions listed in AOCS (American Oil Chemists' Society, 1998), specific gravity of okra seed oil was determined.

\subsection{Efficacy study}

Efficacy trial was accomplished to analyze hypocholesterolemic outcome of okra seed oil. 20 male rats were housed for 30 days in the animal room of National Institute of Food Science and Technology, University of Agriculture, Faisalabad. Temperature and humidity of the animal room was set at $23{ }^{\circ} \mathrm{C}$ and $55{ }^{\circ} \mathrm{C}$ respectively. The experimental rats were adapted by feeding on basal diet for 7 days. During the whole study duration, feed and drink intake of the study subjects were monitored routinely. Weight measurements were also taken before and after the administration of high fat diet. After two weeks, blood serum tests of overnight fasted rats were performed and statistical tool was applied to measure any change in the lipid profile. Animal diet plan is given in Table 1 .

\subsection{Physical parameters}

The following parameters were considered.

\section{Feed and drink intake}

During the whole study time, the net feed intake of rats was calculated routinely. Similarly, food intake of individual rat was estimated everyday by the method explained in Green et al. (2013).

\section{Body weight}

The weight gain of experimental rats was examined after every week throughout the study period.

\section{Serum separation}

Serum was separated from the blood samples based on the method of Sabitha et al. (2011).

\subsection{Efficacy tests}

\section{Total cholesterol}

Total cholesterol was examined by the methods of Sabitha et al. (2011).

\section{Triglycerides}

Triglycerides were examined according to the method of Dubey \& Mishra (2017).

\section{High density lipoproteins}

High density lipoproteins were observed by the method as described by Mekonnen et al. (2018).

Table 1. Animal diet plan.

\begin{tabular}{cl}
\hline Groups & \multicolumn{1}{c}{ Treatments } \\
\hline $\mathrm{G}_{\mathrm{o}}$ & Normal rats \\
$\mathrm{G}_{1}$ & Hypercholesterolemic rats \\
$\mathrm{G}_{2}$ & Hypercholesterolemic rats $+6 \%$ diet containing okra seed oil \\
$\mathrm{G}_{3}$ & Hypercholesterolemic rats $+12 \%$ of diet containing okra seed oil \\
$\mathrm{G}_{4}$ & $\begin{array}{l}\text { Hypercholesterolemic rats }+18 \% \text { of diet containing okra } \\
\text { seed oil }\end{array}$ \\
\hline
\end{tabular}

$G_{0}:$ Normal rats; $G_{1}:$ Hypercholesterolemic rats; $G_{2}:$ Hypercholesterolemic rats $+6 \%$ of diet containing okra seed oil; $\mathrm{G}_{3}$ : Hypercholesterolemic rats $+12 \%$ of diet containing okra seed oil; $\mathrm{G}_{4}:$ Hypercholesterolemic rats $+18 \%$ of diet containing okra seed oil. 


\section{Low density lipoproteins}

Low density lipoproteins were studied by the method of Abolhassani et al. (2017).

\subsection{Statistical analysis}

The data was collected from the experimental study and shifted to statistical analysis to determine the level of significance.

\section{Results and discussion}

The results of different parameters are described here with relevant discussion.

\subsection{Proximate analysis of okra seeds}

The proximate analysis was used to estimate the characteristics of okra seeds. Purposely, okra seed powder was examined for following parametrs.

\section{Moisture content in okra seeds}

Moisture content reported in local okra variety of punjab; named Sabaz pari was $7.24 \%$ during the study. The results were analoged to the study of Çalışır et al. (2005), who compared the proximate profiling and physiochemical properties of two varieties of okra; named as Punjab 8 and Sabaz pari. The moisture content of seed powder of Sabaz pari calculated was 7.26\%.

\section{Crude protein content}

The $\%$ crude protein of both okra seed powder examined in the current study was $20.1 \%$. The outcomes were similar to the study of Çalışır et al. (2005) compared the proximate profiling and physiochemical properties of two varieties of okra; named as Punjab 8 and Sabaz pari. The protein content of seed powder of sabaz pari calculated was $20.00 \%$.

\section{Crude fat content}

The $\%$ crude fat of okra seed powder calculated in the existing study was $11.75 \%$. The value of crude fat was comparable with Çalışır et al. (2005).

\section{Crude fiber content in okra seeds}

The fiber content of okra seed of local variety was $29.54 \%$. Similarly, the fiber content of okra seeds was studied in the study of Soares et al. (2012). The percentage of fiber in the okra seeds was reported as $30.81 \%$, which was greater than the fiber content calculated in the present study. The results of Proximate analysis of okra seeds and physiochemical analysis of okra seed oil are given in Table 2 .

\subsection{Chemical analysis and properties of okra seed oil}

Okra seeds from Greece are a potential source of oil, with concentrations varying from $15.9 \%$ to $20.7 \%$. Of the fatty acid chains that compose okra seed oil, high linoleic acid chain content was observed at $44.48 \%$, palmitic acid was $28.74 \%$, and oleic acid
Table 2. Proximate analysis of okra seeds and physiochemical analysis of okra seed oil

\begin{tabular}{lr}
\hline & Proximate assay of okra seeds \\
\hline Parameter & Means in $\% \pm$ SD \\
Moisture & $7.2 \% \pm 0.01$ \\
Crude protein & $20.1 \% \pm 0.2$ \\
Crude fat & $11.75 \% \pm 0.01$ \\
Crude fiber & $29.54 \% \pm 0.02$ \\
\hline & Chemical analysis of okra seed oil \\
\hline Sponification value & $180.66 \mathrm{mg} \mathrm{KOH} / \mathrm{g} \pm 1.52$ \\
Acid vlaue & $3.45 \mathrm{mg} \mathrm{KOH} / \mathrm{g} \pm 0.03$ \\
Peroxide value & $7.3 \mathrm{meq} / \mathrm{kg} \pm 0.02$ \\
Specific gravity & $0.91 \pm 0.01$ \\
\hline
\end{tabular}

was $20.38 \%$ (András et al., 2005). Gas liquid chromatography exposed that the main fatty acid was linoleic acid (34.89-44\%), palmitic acid (25.2-28.3\%) and oleic acid (21.9-24.08\%). Oleic and linoleic acids (omega 6) are among the fatty acids that have a protecting effect on the health of humans. Okra seed oil showed similarities to certain oils commercially used for their oleic acid content (corn: $24.8 \%$; linseed: $18.9 \%$; poppy seed: $22.3 \%$; soybean: 23.2\%; sunflower seed: $17.7 \%$; walnut kernel: $18.5 \%$ ) and linoleic acid (cottonseed: $57.4 \%$; soybean: $56.2 \%$; walnut kernel: $56.0 \%$ ).

Saponification value

The saponification value of okra seed oil calculated in the present study was $180.66 \pm 1.52 \mathrm{mg} \mathrm{KOH} / \mathrm{g}$ of oil. Various previous studies analyzed the chemical properties of seed oil of different varieties of okra. In a study of Rafieian-Kopaei et al. (2013), the chemical analysis of oil obtained from two different varieties of okra was studied. The saponification value of seed oil obtained from white velvet variety of okra was calculated as 194.5, while that of Perkins Mammoth Long Pod was 192.7.

\section{Peroxide value}

The peroxide value of okra seed oil calculated in the present study was $7.3 \pm 0.02 \mathrm{meq} / \mathrm{kg}$ of oil. The peroxide value (meq of $\mathrm{O}_{2}$ per $\mathrm{kg}$ of oil) recovered from supercritical carbon dioxide extraction was $3.32,3.91$ of solvent extracted okra seed oil while 12.77 of screw pressed okra seed oil (Dong et al., 2014).

\section{Specific gravity}

The specific gravity of okra seed oil evaluated in the present study was $0.91 \pm 0.01$. The specific gravity of okra seed oil calculated in the study of Mohapatra et al. (2007) on triglyceride composition of Hibiscus esculentus seed oil was 0.9135 at 24 degree centigrade.

\section{Acid value}

The acid value in current study was in accordance with the study of Çalışır et al. (2005), in which inter-varietal variation in 
the composition of okra seed oil was evaluated. Two varieties of okra named Sabaz pari and Punjab 8 were compared in terms of their physiochemical properties. The acid value of two varieties was in the range of 3.49-4.67 ( $\mathrm{mg}$ of $\mathrm{KOH} \mathrm{g}^{-1}$ of oil).

\subsection{Bio-efficacy study}

Depending on diet, the efficacy plan was categorized into five groups, negative control, positive control, treatment group $\left(\mathrm{G}_{2}\right)$, treatment group $\left(G_{3}\right)$, and treatment group $\left(G_{4}\right)$. Negative control group was fed with normal diet, positive control group was fed with high fat diet to induce hypercholesterolemia, treatment $\left(G_{2}\right)$, was fed with high fat diet $+6 \%$ okra seed oil, treatment $\left(G_{3}\right)$ was fed with high fat diet $+12 \%$ okra seed oil, and treatment $\left(G_{4}\right)$ was fed with high fat diet $+18 \%$ okra seed oil. The trial was carried out for 30 days to determine the anti-hypercholesterolemic effect of okra seed oil. The body weight was calculated on weekly basis, while feed and drink intakes were calculated on daily basis. The serum was collected in start and at the end of the trail to analyze total cholesterol and related parameters of lipid profile.

\section{Body weight}

In this research work the impact of okra seed oil was evaluated on body weight of male Sprague Dowley rats including normal, hypercholesterolemic groups and diseased groups with $6 \%, 12 \%$ and $18 \%$ okra seed oil as treatment respectively. It is deduced from the mean squares (Table 3 ) that treatment imparts significant effect on all groups except positive and negative control groups. However, positive and negative control groups had followed by weight gain with respect to days. At the end of the trail, the means for body weight was $153.45 \pm 1.877$ of negative control group. The means of body weight in positive control group was 157.2 \pm 3.63 , which was higher than negative control group because of the administration of high fat diet. In treatment groups; the means of cholesterol was $155.55 \pm 3.17$, $156.85 \pm 3.78$ and $156.75 \pm 4.58$ respectively.

\section{Feed intake}

Feed status of all rats was noted daily. The analysis of variance for feed intake showed the non-significant effect of treatments on the feed intake and significant effect on the study interval (Table 3). Throughout the efficacy trial of 30 days, the mean difference of treatments showed non-significant effect on the routine feed intake of the hypercholesterolemic rats. The means of the feed intake showed the highest value in G1 group $(15.9 \pm 1.68 \mathrm{~g} / \mathrm{rat} /$ day $)$. While means of feed intake of control group Go was $(14.5 \pm 0.84 \mathrm{~g} / \mathrm{rat} /$ day $), \mathrm{G} 2(15.6 \pm 1.69 \mathrm{~g} / \mathrm{rat} /$ day $)$ and G3 (14.7 $\pm 1.65 \mathrm{~g} / \mathrm{rat} /$ day $)$.

\section{Drink intake}

The rat's drink intake was noted daily throughout animal study. The mean square for drink intake in the first week were noted as $18.5 \pm 1.29,18.5 \pm 1.29,18.82 \pm 0.81,18 \pm 1.41,18.75 \pm 1.25$ for G0, G1, G2 and G3 and G4 respectively while at the final week $18.62 \pm 1.108,18.5 \pm 1.29,19 \pm 1.08,18.12 \pm 1.16$ and $18.87 \pm 1.31 \mathrm{~mL} / \mathrm{rat} /$ day for the respective groups. The mean comparisons for the drink intake in the (Table 3) showed that the treatments have no definite effect on the increase in the drink status of rats with the increase in the number of days. The research work of Cheng et al. (2017) explained the link between the high fat diet and the drink intake of post weaning and adult rats.

\section{Anti-hypercholesterolemic potential}

The anti-hypercholesterolemic effect of okra seed oil is because of polyunsaturated fatty acids mainly linoleic acid that has the capability to start the relocate of cholesterol in the direction of bile acid. Changes in body weight (a), feed intake (b), LDL (c), drink intake (d), cholesterol (e), HDL (f) of different groups of rats are demonstrated in Figure 1.

\section{Cholesterol}

In current study work the impact of okra seed oil was evaluated on changes in serum lipid profiles of male Sprague Dowley rats including normal, hypercholesterolemic groups and diseased groups with $6 \%, 12 \%$ and $18 \%$ okra seed oil respectively with particular mention to cholesterol, low density lipoprotein, high density lipoprotein and triglycerides. It is concluded from the mean squares (Table 3 ) that treatment imparts significant effect on all groups except positive and Go. At the end of the trail, the means for cholesterol was $88.25 \pm 2$ of negative control group. The means of cholesterol in G1 was increased to $91.25 \pm 2.49$ and in treatment groups; the means of cholesterol was $90.5 \pm 2.39,89.25 \pm 3.88$ and $87.25 \pm 4.07$ respectively. With special reference to different day's intervals, the means of cholesterol for all groups was $88.9 \pm 2.22$ on Day 1 . Similarly, on day 7, the means of cholesterol for all groups was $91.25 \pm 2.76$ and on Day 28, the means of cholesterol for all groups was $87.75 \pm 3.86$. Although all the treatments of okra seed oil significantly reduced the serum cholesterol levels in Sprague Dowley rats, but the best hypocholesterolemic results were given by $18 \%$ okra seed oil in the daily diet of hypercholesterolemic rats. At the end of the trail 6\% okra seed oil treatment reduced cholesterol levels up-to $90.5 \pm 2.39$ while $12 \%$ okra seed oil treatment reduced cholesterol levels up-to $89.25 \pm 3.88$ and $18 \%$ okra seed oil treatment reduced cholesterol levels up-to $87.25 \pm 4.07$.

\section{Low density lipoproteins (LDL)}

In this research work the impact of okra seed oil was evaluated on changes in serum lipid profiles of male Sprague Dowley rats including normal, hypercholesterolemic groups and diseased groups with $6 \%, 12 \%$ and $18 \%$ okra seed oil respectively with special mentioning of cholesterol, low density lipoprotein, high density lipoprotein and triglycerides. Variance showed the mean comparisons of serum LDL cholesterol levels with respect to treatments and days (Table 3 ). In the group $G_{0}$ the means of serum LDL cholesterol levels was $44.25 \pm 1.54$, which was increased to $45.4 \pm 3.62$ in positive control group G1, due to giving high fat diet. In treatment groups, the means of serum LDL cholesterol as a result of inducing hypercholesterolemia in subjects and followed by treatments of $6 \%, 12 \%$ and $18 \%$ 
Table 3. Mean comparison for body weight gain (g), feed intake(g/day), drink intake (mL/day), cholesterol (g/dL), LDL (g/dL), HDL (g/dL), and triglycerides $(\mathrm{g} / \mathrm{dL})$.

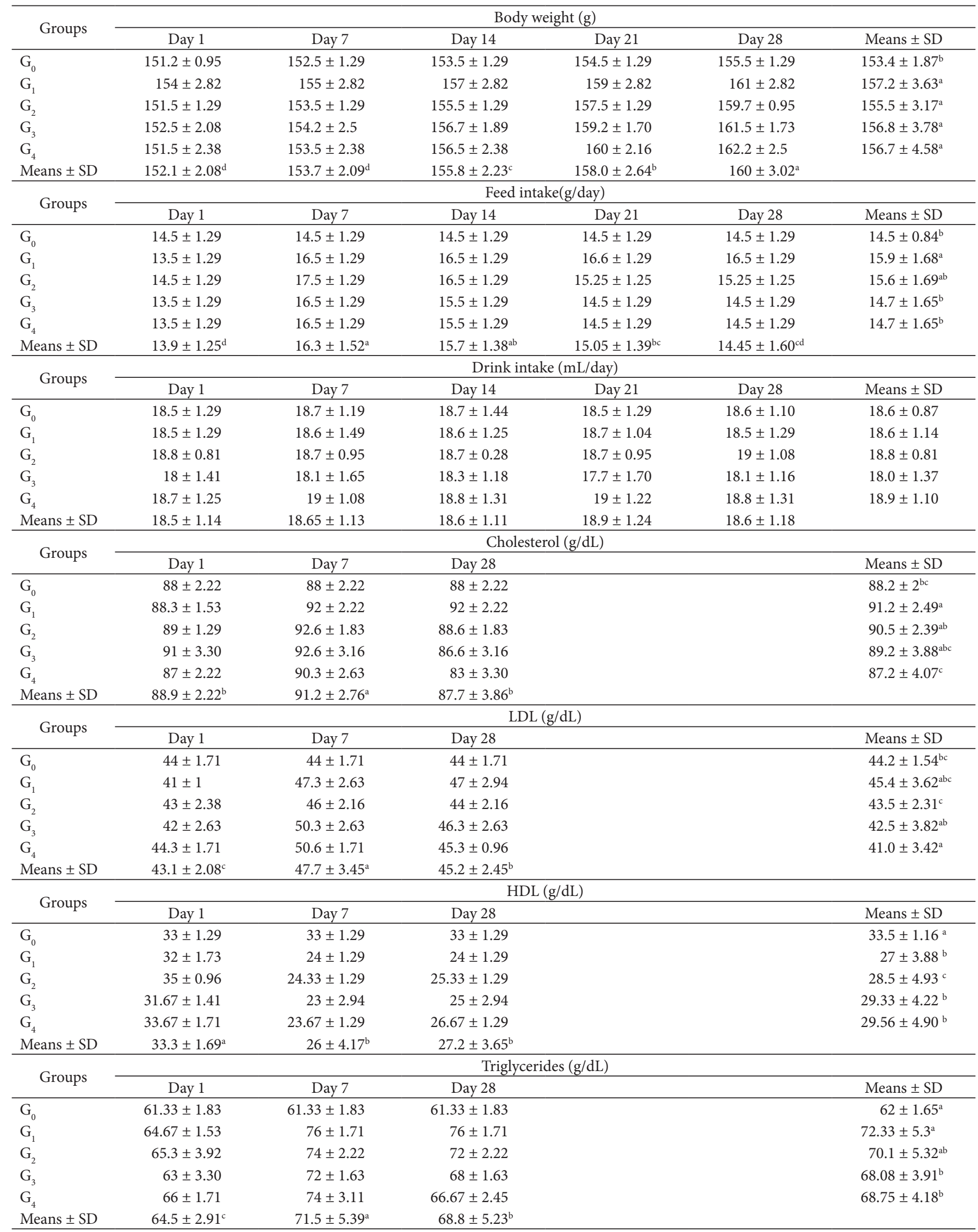



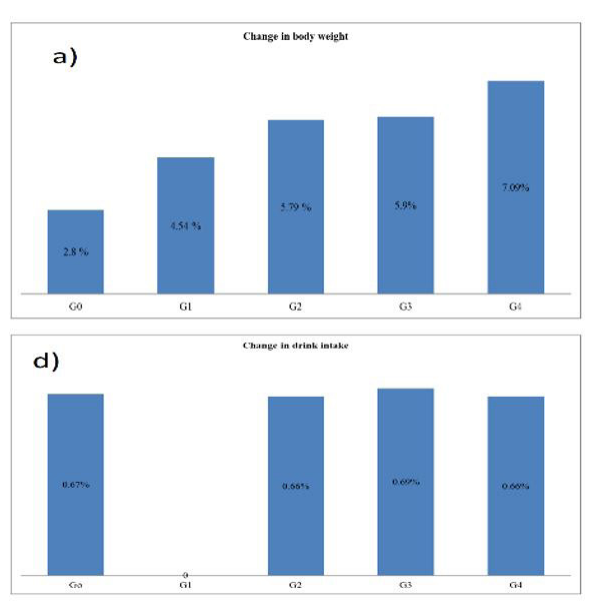
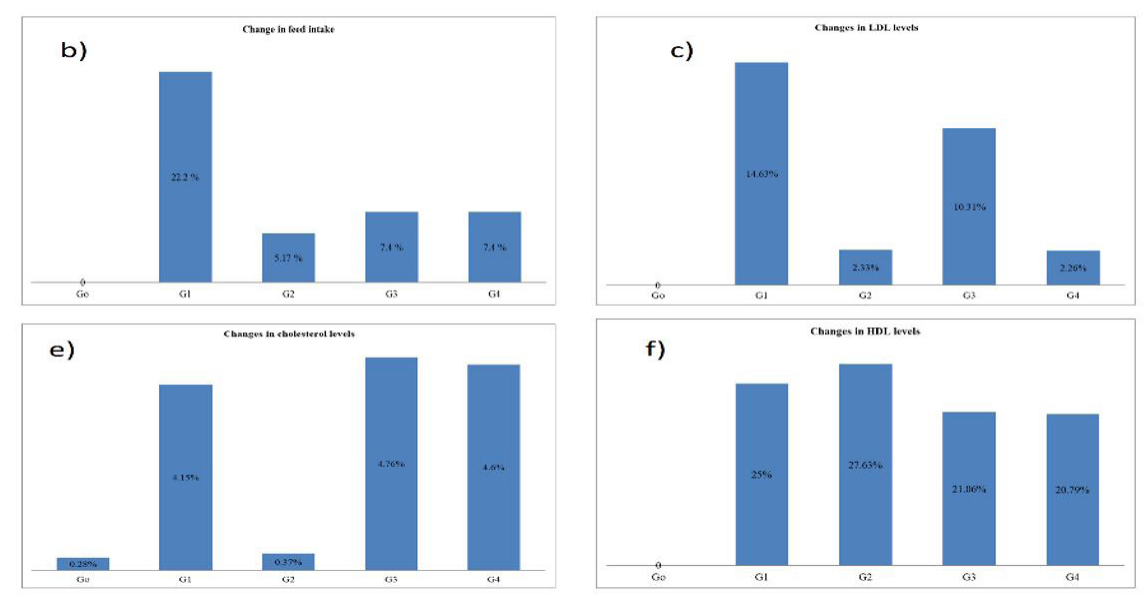

Figure 1. Changes in body weight (a), feed intake (b), LDL (c), drink intake (d), cholesterol (e), HDL (f) of different groups of rats.

okra seed oil respectively were $43.5 \pm 2.31,42.5 \pm 3.82$ and $41.08 \pm 3.42$. The study of Dubey \& Mishra (2017) concluded the reducing effect of okra seed on LDL cholesterol after performing bio- efficacy study on male albino rats for 42 days.

After inducing hypercholesterolemia in rats in the duration of 30 days, dried okra seed powder at a dose of 250 and $500 \mathrm{mg} / \mathrm{kg}$ body weight of rats, was given to rats for 42 days. The results of the respective study showed 30\% significant reduction of serum LDL cholesterol levels in rats.

\section{High density lipoproteins (HDL)}

In this research work the impact of okra seed oil was evaluated on changes in serum lipid profiles of male Sprague Dowley rats including normal, hypercholesterolemic groups and diseased groups with $6 \%, 12 \%$ and $18 \%$ okra seed oil respectively with special reference to cholesterol, low density lipoprotein, high density lipoprotein and triglycerides. The variance showed the mean comparisons of serum HDL cholesterol levels with respect to treatments and days (Table 3). In the group $\mathrm{G}_{0}$ the means of serum HDL cholesterol levels was $33.5 \pm 1.16$, which was decreased to $27 \pm 3.88$ in positive control group G1, due to administration of high fat diet. In treatment groups, the means of serum HDL cholesterol as a result of inducing hypercholesterolemia in subjects and followed by treatments of $6 \%, 12 \%$ and $18 \%$ okra seed oil respectively were $28.5 \pm 4.93,29.33 \pm 4.22$ and $29.56 \pm 4.90$.

\section{Triglycerides}

In this research work the impact of okra seed oil was evaluated on changes in serum lipid profiles of male Sprague Dowley rats including normal, hypercholesterolemic groups and diseased groups with $6 \%, 12 \%$ and $18 \%$ okra seed oil respectively with particular reference to high density lipoprotein, cholesterol, low density lipoprotein and triglycerides. In the group $\mathrm{G}_{0}$ the means of serum triglyceride levels was $62 \pm 1.65$, which was increased to $72.33 \pm 5.3$ in positive control group $\mathrm{G}_{1}$, due to high fat diet. In treatment groups, the means of serum triglyceride levels as a result of inducing hypercholesterolemia in Sprague Dowley rats, and followed by treatments of $6 \%, 12 \%$ and $18 \%$ okra seed oil respectively were $70.1 \pm 5.32,68.08 \pm 3.91$ and $68.75 \pm 4.18$. The results showed significant impact of days and treatments on serum triglyceride levels of Sprague Dowley rats. Abolhassani et al. (2017) assessed the upshot of mucilage of okra pods on the raised serum lipid profile of Ratus Norvegicus by comparing the effect with garlic and lemon mixture. The study was performed on 34 Ratus Norvegicus. 25\% significant reduction was observed triglyceride levels of hypercholesterolemic rats receiving $10 \%$ okra mucilage for 3 weeks.

\section{Conclusion}

Okra is gaining importance due to its therapeutic activities and also known as functional food owing to its pharmacological properties mainly anti-diabetic, anti-hypercholesterolemic and anti- obesity activities. Hypercholesterolemia leads to various complications due to oxidative stress and elevated cholesterol level in the blood. Purposely, present study was conducted to check the anti-hypercholesterolemic properties of okra seed oil in hypercholesterolemic rats. Okra seeds were subjected for proximate analysis, while seed oil was subjected for evaluating chemical analysis. Mechanical extraction method was used for extracting the oil from seeds of okra. A bio-efficacy study was organized to calculate the effects of okra seed oil on hypercholesterolemia. Results of proximate analysis showed that okra seeds contained $7.2 \%$ moisture, $20.1 \%$ protein, $11.75 \%$ fat, $29.54 \%$ fiber content. Physiochemical analysis of okra seed oil showed that saponification value of okra seed oil was 180.66 , acid value was 3.45 , peroxide value was 7.3 and specific gravity of 0.91 . In bio-efficacy study five different groups of rats were designed. $G_{0}$ normal rats, $G_{1}$ positive control group, $G_{2}$ hypercholesterolemic rats $+6 \%$ okra seed oil, $\mathrm{G}_{3}$ hypercholesterolemic rats $+12 \%$ okra seed oil, $\mathrm{G}_{4}$ hypercholesterolemic rats $+18 \%$ okra seed oil. Okra seed oil showed significant effect on body weight. Maximum weight increase was examined in group receiving $18 \%$ okra seed oil. Moreover, treatments have non-significant effect on the feed intake and drink intake of rats. Decreasing pattern was observed for triglycerides, LDL and cholesterol. While increasing pattern was observed with HDL due to the administration of okra seed oil. Concisely, okra (Abelmoschus esculentus) seed oil has been proved as advantageous in reducing hypercholesterolemia. Okra 
seed oil plays crucial role in maintenance of lipid profile in the body. Conclusively, it was concluded from present study that okra possess essential health benefits.

\section{References}

Abolhassani, M., Eftekhari, N., Basirinezhad, M. H., \& Norouzi, P. (2017). Comparing the effect of garlic and lemon mixture with mucilage of okra pods to prevent the increase of plasma lipids in ratus norvegicus alluvia. International Journal of Health Studies, 3(2), 25-28.

American Oil Chemists' Society - AOCS. (1998). Official Methods and Recommended Practices of the American Oil Chemists' Society. Champaign: AOCS Press.

András, C. D., Simándi, B., Örsi, F., Lambrou, C., Missopolinou-Tatala, D., Panayiotou, C., Domokos, J., \& Doleschall, F. (2005). Supercritical carbon dioxide extraction of okra (Hibiscus esculentus L.) seeds. Journal of the Science of Food and Agriculture, 85(8), 1415-1419. http://dx.doi.org/10.1002/jsfa.2130.

Association of Official Analytical Chemists - AOAC. (2016). The Official Methods of Analysis of AOAC International. The Association of Official Analytical Chemists (20 ${ }^{\text {th }}$ ed.). Rockville, MD, USA: AOAC.

Çalışır, S., Özcan, M., Hacıseferoğulları, H., \& Yıldız, M. U. (2005). A study on some physico-chemical properties of Turkey okra (Hibiscus esculenta L.) seeds. Journal of Food Engineering, 68(1), 73-78. http:// dx.doi.org/10.1016/j.jfoodeng.2004.05.023.

Cheng, H. S., Ton, S. H., Phang, S. C. W., Tan, J. B. L., \& Abdul Kadir, K. (2017). Increased susceptibility of post-weaning rats on high-fat diet to metabolic syndrome. Journal of Advanced Research, 8(6), 743752. http://dx.doi.org/10.1016/j.jare.2017.10.002. PMid:29062573.

Dong, Z., Zhang, J. G., Tian, K. W., Pan, W. J., \& Wei, Z. J. (2014). The fatty oil from okra seed: supercritical carbon dioxide extraction, composition and antioxidant activity. Current Topics in Nutraceutical Research, 12(3), 75.

Dubey, P., \& Mishra, S. (2017). Effect of okra seed in reduction of cholesterol. Journal of Entomology and Zoology Studies, 5(4), 94-97.

Gemede, H. F., Ratta, N., Haki, G. D., Woldegiorgis, A. Z., \& Beyene, F. (2015). Nutritional quality and health benefits of okra (Abelmoschus esculentus): A review. Journal of Food Processing \& Technology, 6(458), 2. http://dx.doi.org/10.4172/2157-7110.1000458.
Green, C. O., Wheatley, A. O., Mcgrowder, D. A., Dilworth, L. L., \& Asemota, H. N. (2013). Citrus peel polymethoxylated flavones extract modulates liver and heart function parameters in diet induced hypercholesterolemic rats. Food and Chemical Toxicology, 51, 306309. http://dx.doi.org/10.1016/j.fct.2012.10.005. PMid:23099503.

Mekonnen, Z., Gebreselema, A., \& Abere, Y. (2018). Effect of locally manufactured niger seed oil on lipid profile compared to imported palm and sunflower oils on rat models. Journal of Lipids, 2018:7846350. http://dx.doi.org/10.1155/2018/7846350. PMid:29854466.

Misra, A., Soares, M. J., Mohan, V., Anoop, S., Abhishek, V., Vaidya, R., \& Pradeepa, R. (2018). Body fat, metabolic syndrome and hyperglycemia in South Asians. Journal of Diabetes and Its Complications, 32(11), 1068-1075. http://dx.doi.org/10.1016/j. jdiacomp.2018.08.001. PMid:30115487.

Mohapatra, M. R., Acharya, P., \& Sengupta, S. (2007). Variability and association analysis in okra. Indian Agriculturist, 51(1/2), 17-26.

Nahar, J., Imam, T., Tickle, K. S., \& Chen, Y. P. P. (2013). Association rule mining to detect factors which contribute to heart disease in males and females. Expert Systems with Applications, 40(4), 10861093. http://dx.doi.org/10.1016/j.eswa.2012.08.028.

Rafieian-Kopaei, M., Asgary, S., Hajian, S., \& Roozbehani, S. (2013). Effect of mucilage extracted from the fruit of Hibiscus esculentus on preventive of increasing glucose and lipid profile of diabetic rats by streptozotocin. Shahrekord University of Medical Sciences Journal, 15(3), 48-55.

Sabitha, V., Ramachandran, S., Naveen, K. R., \& Panneerselvam, K. (2011). Antidiabetic and antihyperlipidemic potential of Abelmoschus esculentus (L.) Moench. in streptozotocin-induced diabetic rats. Journal of Pharmacy \& Bioallied Sciences, 3(3), 397-402. http:// dx.doi.org/10.4103/0975-7406.84447. PMid:21966160.

Soares, G. S. F., Gomes, V. D. M., dos Reis Albuquerque, A., Barbosa Dantas, M., Rosenhain, R., Souza, A. G. D., Persunh, D. C., Gadelha, C. A. A., Costa, M. J. C., \& Gadelha, T. S. (2012). Spectroscopic and thermooxidative analysis of organic okra oil and seeds from Abelmoschus esculentus. The Scientific World Journal, 2012:84747. http://dx.doi.org/10.1100/2012/847471. PMid:22645459.

Topkafa, M. (2016). Evaluation of chemical properties of cold pressed onion, okra, rosehip, safflower and carrot seed oils: triglyceride, fatty acid and tocol compositions. Analytical Methods, 8(21), 4220-4225. http://dx.doi.org/10.1039/C6AY00709K. 